Document downloaded from:

http://hdl.handle.net/10251/146303

This paper must be cited as:

Amat, S.; Argyros, IK.; Busquier Saez, S.; Hernández-Verón, MA.; Martínez Molada, E. (011). On the local convergence study for an efficient k-step iterative method. Journal of Computational and Applied Mathematics. 343:753-761.

https://doi.org/10.1016/j.cam.2018.02.028

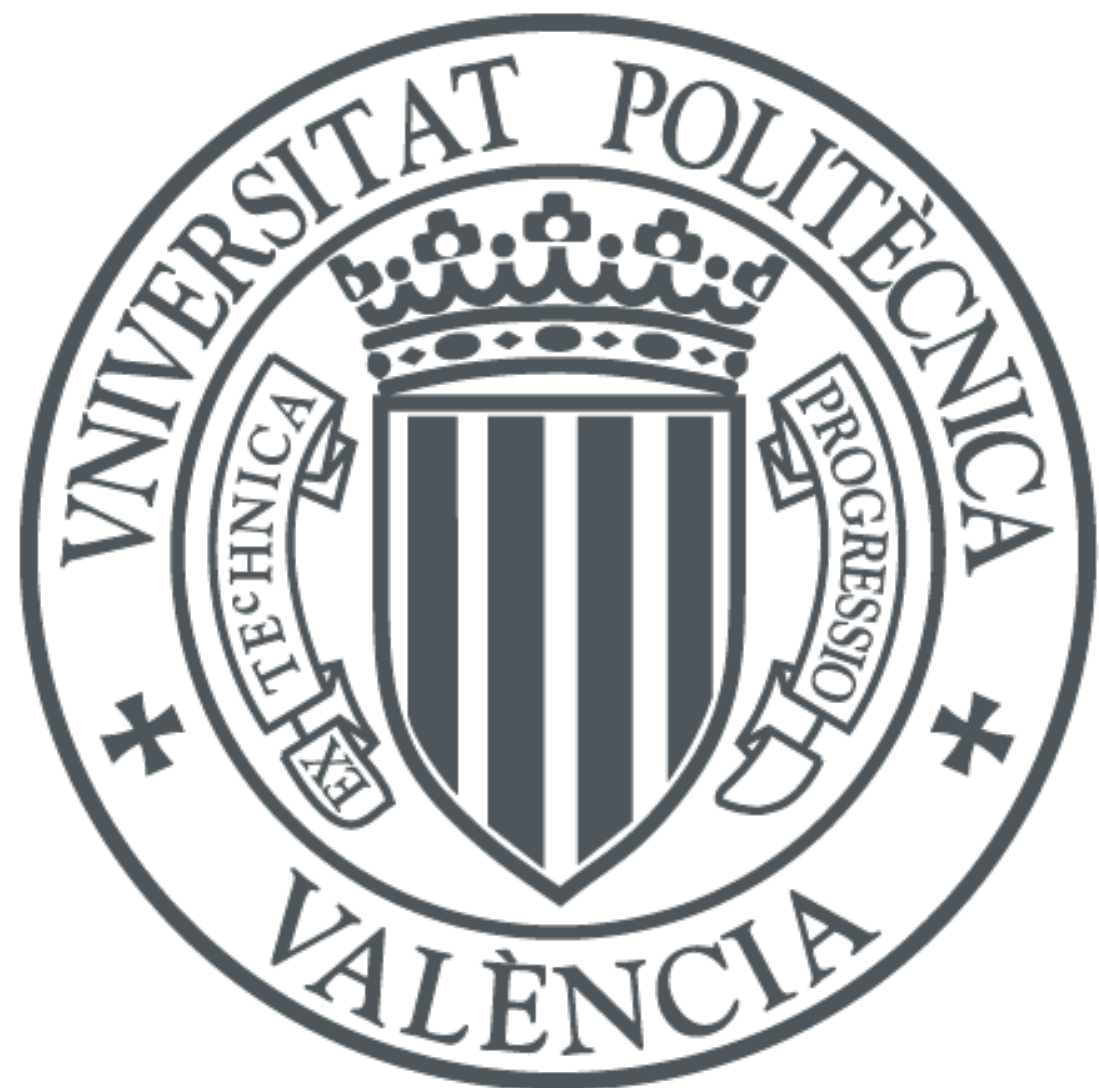

The final publication is available at

https://doi.org/10.1016/j.cam.2018.02.028

Copyright Elsevier

Additional Information 


\title{
On the local convergence study for an efficient k-step iterative method
}

\author{
S. Amat ${ }^{a}$, I.K. Argyros ${ }^{\text {b }}$, S. Busquier ${ }^{\text {a }}$, M. A. Hernández-Verón ${ }^{\text {c }}$, E. Martínez $^{\text {d }}$. \\ ${ }^{a}$ Dpt. Applied Mathematics and Statistic, U.P. Cartagena. \\ Cartagena, Spain. \\ E-mail address: sergio.amat@upct.es; sonia.busquier@upct.es \\ b Dpt. of Mathematical Sciences, Cameron University. \\ Lawton, OK 73505, USA. \\ E-mail address: iargyros@cameron.edu \\ ${ }^{c}$ Dpt. of Mathematics and Computation, University of La Rioja. \\ 26004 Logroño, Spain. \\ E-mail address: mahernan@unirioja.es \\ ${ }^{\mathrm{d}}$ Instituto Universitario de Matemática Multidisciplinar, Universitat Politècnica de València, \\ 46022 Valencia, Spain. \\ E-mail address: eumarti@mat.upv.es
}

\begin{abstract}
This paper is devoted to a family of Newton-like methods with frozen derivatives used to approximate a locally unique solution of an equation. The methods have high order of convergence but only using first order derivatives. Moreover only one LU decomposition is required in each iteration. In particular, the methods are real alternatives to the classical Newton method. We present a local convergence analysis based on hypotheses only on the first derivative. This type of local results were usually proved based on hypotheses on the derivative of order higher than two although only the first derivative appears in these type of methods [2, 22, 24]. We apply these methods to an equation related to the nonlinear complementarity problem. Finally, we find the most efficient method in the family for this problem and we perform a theoretical and a numerical study for it.
\end{abstract}


Keywords: Nonlinear equations, iterative methods, local convergence, order of convergence, efficiency.

2000 Mathematics Subject Classification: 47H99, 65H10.

Research supported in part by Programa de Apoyo a la investigación de la fundación Séneca-Agencia de Ciencia y Tecnología de la Región de Murcia 19374/PI/14, by the project of Generalitat Valenciana Prometeo/2016/089 and the projects MTM2015-64382-P (MINECO/FEDER), MTM2014-52016-C2-1-P and MTM2014-52016-C2-2-P of the Spanish Ministry of Science and Innovation.

\section{Introduction}

The approximation of the solutions of nonlinear equations $F(x)=0$ is a common problem in science and engineering. Let us consider $F: D \subset X \rightarrow Y$ to be a nonlinear operator where $X$ and $Y$ are Banach spaces.

In order to obtain approximations to the solution, we will use iterative methods of the form

$$
x_{n+1}=\Phi\left(x_{n}\right), \quad n \geq 0,
$$

starting with a given initial approximation $x_{0}$ of the solution $x^{*}$, where $\Phi$ is a function defined on a closed subset $\Omega$ of $X$ that maps $\Omega$ into itself.

The choice of a method for approximating $x^{*}$ usually depends on its efficiency, which links the speed of convergence (order of convergence) of the method to its computational cost. For one-point iterative methods without memory, it is known that the order of convergence $\nu$ is a natural number and the methods depend explicitly on the first $\nu-1$ derivatives of the function involved in the equation. On the other hand, the computational cost increases as it is necessary to calculate the successive derivatives of the function involved in the algorithm of a method. In this paper, we are interested in numerical methods that avoid the expensive computation of the derivatives of the function $F$ at each step.

We start with the iterative process:

$$
\left\{\begin{array}{l}
x_{0} \in D, \\
y_{n}=x_{n}+\Gamma_{n} F\left(x_{n}\right), \\
x_{n+1}=y_{n}-\Gamma_{n} F\left(y_{n}\right), \quad n \geqslant 0 .
\end{array}\right.
$$

where $\Gamma_{n}=F^{\prime}\left(x_{n}\right)^{-1}$.

This method has order of convergence three. In [2], we proved that this iterative process (2) seems to have simpler dynamics than if we consider the different modifications shown by means of Newton's method [5]. This fact tells us that, from a numerical point of view, the implementation of iterative process (2) is more favorable than if we consider $k$-step Newton's method with frozen derivative, given by Traub's method [24]. In any case, in this paper, we will consider an extra parameter in order to analyze the different possibilities. 
If we apply many steps, keeping the derivative frozen, we can obtain

$$
\left\{\begin{array}{l}
x_{0} \in D \\
x_{n}^{(1)}=x_{n}^{(0)}+\alpha \Gamma_{n} F\left(x_{n}^{(0)}\right) \\
x_{n}^{(2)}=x_{n}^{(1)}-\Gamma_{n} F\left(x_{n}^{(1)}\right) \\
\quad \vdots \\
x_{n}^{(k-1)}=x_{n}^{(k-2)}-\Gamma_{n} F\left(x_{n}^{(k-2)}\right), \\
x_{n}^{(k)}=x_{n}^{(k-1)}-\Gamma_{n} F\left(x_{n}^{(k-1)}\right), \quad n \geqslant 0
\end{array}\right.
$$

where $\alpha \in \mathbb{R}, x_{n}=x_{n}^{(0)}$ and $x_{n+1}=x_{n}^{(k)}$.

This method has order of convergence $k+1$, with $k \geq 2$ for $\alpha= \pm 1$. It was presented by us in [2] from a dynamical point of view. The main advantage of these methods is that there is not need to evaluate any high order Fréchet derivative, having the same matrix in each k-step. In particular only one LU decomposition is required in each iteration. This type of methods appear in many applications where the authors heuristically choose a given number of steps with frozen derivatives (see for instance this incomplete list of Refs. $[12,14,15,17,20])$.

The study about convergence matter of iterative procedures is usually based on two types: semi-local and local convergence analysis. The semi-local convergence matter is, based on the information around an initial point, to give conditions ensuring the convergence of the iterative procedure; while the local one is, based on the information around a solution, to find estimates of the radii of convergence balls. In [3] we present a semilocal convergence analysis, for $\alpha=1$, and a general study on the efficient of the different methods in the family.

In the present paper, we first develop a local convergence analysis based on hypotheses only on the first derivative for $\alpha \in \mathbb{R}$. Usually, this type of local results are proved based on hypotheses on the derivative of order higher than two although only the first derivative appears in these type of methods $[2,22,24]$. Next, we apply these methods to an equation related to the nonlinear complementarity problem [19]. Following [5], we find the most efficient method in the family for this problem using the efficiency index and the computational efficiency. In [5] we only studied the case $\alpha=-1$ (Traub's method [24]). Finally, we verify the hypotheses for the local convergence for a particular problem and we approximate the solution using the proposed methods.

Related studies concerning $k$-step Newton-type methods can be found in this incomplete list $[1,4,7,8,9,10,11,13,18,21]$.

\section{Local convergence analysis}

We present the local convergence analysis of method (3) in this section. We only use hypotheses on the first derivative. It is convenient for our local convergence analysis that follows to introduce some functions and parameters. 
Let $L_{0}>0, L>0, M \geq 1, \alpha \in \mathbb{R}$ and $k \in \mathbb{N}$ be given parameters.

Define functions $g_{1}, p_{1}$ and $\bar{p}_{1}$ on the interval $\left[0, \frac{1}{L_{0}}\right)$ by

$$
g_{1}(t)=\frac{1}{2\left(1-L_{0} t\right)}(L t+2|1+\alpha| M)
$$

$p_{1}(t)=L_{0} g_{1}(t) t$ and $\bar{p}_{1}(t)=p_{1}(t)-1$.

We have that $\bar{p}_{1}(0)=-1<0$ and $\bar{p}_{1}(t) \rightarrow+\infty$ as $t \rightarrow{\frac{1}{L_{0}}}^{-}$. Then, it follows from the intermediate value theorem that function $\bar{p}_{1}$ has zeros in the interval $\left(0, \frac{1}{L_{0}}\right)$. Denote by $r_{\bar{p}_{1}}$ the smallest such zero.

Define the functions $g_{2}, \bar{g}_{2}, p_{2}$ and $\bar{p}_{2}$ on the interval $\left[0, r_{\bar{p}_{1}}\right)$ by

$$
g_{2}(t)=\left(\frac{L g_{1}(t)}{2\left(1-L_{0} g_{1}(t) t\right)}+\frac{L_{0} M\left(1+g_{1}(t)\right)}{\left(1-L_{0} g_{1}(t) t\right)\left(1-L_{0} t\right)}\right) g_{1}(t) t
$$

$\bar{g}_{2}(t)=g_{2}(t)-1, p_{2}(t)=L_{0} g_{2}(t) t$ and $\bar{p}_{2}(t)=p_{2}(t)-1$.

We have that $\bar{g}_{2}(0)=\bar{p}_{2}(0)=-1<0$, and $\bar{g}_{2}(t) \rightarrow+\infty, \bar{p}_{2}(t) \rightarrow+\infty$ as $t \rightarrow r_{\bar{p}_{1}}^{-}$. By the intermediate value theorem, we deduce the existence of $r_{\bar{g}_{2}}$ and $r_{\bar{p}_{2}}$ the smallest zeros of functions $\bar{g}_{2}(t)$ and $\bar{p}_{2}(t)$ respectively on the interval $\left(0, r_{\bar{p}_{1}}\right)$.

Moreover, using induction, define functions $g_{i}, \bar{g}_{i}, p_{i}$ and $\bar{p}_{i}$ on the interval $\left[0, r_{\bar{p}_{i-1}}\right)$ by

$$
g_{i}(t)=\left(\frac{L g_{i-1}(t)}{2\left(1-L_{0} g_{i-1}(t) t\right)}+\frac{L_{0} M\left(1+g_{i-1}(t)\right)}{\left(1-L_{0} g_{i-1}(t) t\right)\left(1-L_{0} t\right)}\right) g_{i-1}(t) t
$$

$\bar{g}_{i}(t)=g_{i}(t)-1, p_{i}(t)=L_{0} g_{i}(t) t$ and $\bar{p}_{i}(t)=p_{i}(t)-1$, for each $i=2,3, \ldots, k$.

We have that $\bar{g}_{i}(0)=\bar{p}_{i}(0)=-1<0$, and $\bar{g}_{i}(t) \rightarrow+\infty, \bar{p}_{i}(t) \rightarrow+\infty$ as $t \rightarrow r_{\bar{p}_{i-1}}^{-}$. Using again the intermediate value theorem, we deduce the existence of $r_{\bar{g}_{i}}$ and $r_{\bar{p}_{i}}$ the smallest zeros of functions $\bar{g}_{i}(t)$ and $\bar{p}_{i}(t)$ respectively on the interval $\left(0, r_{\bar{p}_{i-1}}\right)$.

Furthermore, define parameters $r, \tilde{r}$ and $R$ by

$$
\begin{gathered}
r=\min _{i=2,3, \ldots, k}\left\{r_{\bar{g}_{i}}, r_{\bar{p}_{k}}\right\}, \\
\tilde{r}=g_{1}(r) r, \\
R=\max \{r, \tilde{r}\} .
\end{gathered}
$$

Then, for each $t \in[0, r)$,

$$
0 \leq g_{i}(t)<1 \quad \text { for } \quad i=2,3, \ldots, k \text {. }
$$

Denote by $U(x, \rho)$ and $\bar{U}(x, \rho)$ the open and closed balls in $X$ respectively with center $x \in X$ and radius $\rho>0$.

Next, we present our local convergence analysis using the preceding notation. 
Theorem 1 Let $F: D \subseteq X \rightarrow Y$ be a Fréchet differentiable operator. Suppose:

There exists $x^{*} \in D, L_{0}>0, L>0, M \geq 1, k \in \mathbb{N}, \alpha \in \mathbb{R}$ such that

$$
\begin{gathered}
F\left(x^{*}\right)=0, F^{\prime}\left(x^{*}\right)^{-1} \in \mathrm{L}(Y, X), \\
\left\|F^{\prime}\left(x^{*}\right)^{-1}\left(F^{\prime}(x)-F^{\prime}\left(x^{*}\right)\right)\right\| \leq L_{0}\left\|x-x^{*}\right\|, \quad \text { for all } x \in D, \\
\left\|F^{\prime}\left(x^{*}\right)^{-1}\left(F^{\prime}(x)-F^{\prime}(y)\right)\right\| \leq L\|x-y\|, \quad \text { for all } x, y \in D_{0}=D \bigcap U\left(x^{*}, \frac{1}{L_{0}}\right), \\
\left\|F^{\prime}\left(x^{*}\right)^{-1} F^{\prime}(x)\right\| \leq M, \text { for all } x \in D_{0},
\end{gathered}
$$

and

$$
\bar{U}\left(x^{*}, R\right) \subseteq D
$$

where $R$ is defined by (6).

Then the sequence $\left\{x_{n}\right\}$ generated for $x_{0} \in U\left(x^{*}, r\right) \backslash\left\{x^{*}\right\}$ by the method (3) is well defined, remains in $U\left(x^{*}, r\right)$ and converges to $x^{*}$. Moreover, the following estimates hold

$$
\begin{gathered}
\left\|x_{n}^{(1)}-x^{*}\right\| \leq g_{1}\left(\left\|x_{n}-x^{*}\right\|\right)\left\|x_{n}-x^{*}\right\| \leq \tilde{r}, \\
\left\|x_{n}^{(i)}-x^{*}\right\| \leq g_{i}\left(\left\|x_{n}-x^{*}\right\|\right)\left\|x_{n}-x^{*}\right\| \leq r,
\end{gathered}
$$

for each $i=2,3, \ldots, k-1$, and

$$
\left\|x_{n+1}-x^{*}\right\| \leq g_{k}\left(\left\|x_{n}-x^{*}\right\|\right)\left\|x_{n}-x^{*}\right\|<\left\|x_{n}-x^{*}\right\|,
$$

where the 'g' functions, and the parameters $r, \tilde{r}$ are defined above this Theorem, see (4)-(7).

Furthermore, for $T \in\left[r, \frac{2}{L_{0}}\right)$ the limit point $x^{*}$ is the only solution of the equation $F(x)=0$ in $\bar{U}\left(x^{*}, T\right) \bigcap D$.

\section{Proof}

We shall show the estimates using mathematical induction.

Let $x \in U\left(x^{*}, R\right)$. Then using (9) we have that

$$
\left\|F^{\prime}\left(x^{*}\right)^{-1}\left(F^{\prime}(x)-F^{\prime}\left(x^{*}\right)\right)\right\| \leq L_{0}\left\|x-x^{*}\right\|<L_{0} R<1 .
$$

It follows from (16) and the Banach lemma of invertible operators [16] that $F^{\prime}(x)^{-1} \in$ $L(X, Y)$ and

$$
\left\|F^{\prime}(x)^{-1} F^{\prime}\left(x^{*}\right)\right\| \leq \frac{1}{1-L_{0}\left\|x-x^{*}\right\|} .
$$

In particular, (17) holds for $x=x_{0}$, since $x_{0} \in U\left(x^{*}, R\right) \backslash\left\{x^{*}\right\}$ and $x_{0}^{(1)}$ is well defined for the first subset of the method $(n=0)$.

By (8) we can write

$$
F\left(x_{0}\right)=F\left(x_{0}\right)-F\left(x^{*}\right)=\int_{0}^{1} F^{\prime}\left(x^{*}+\theta\left(x_{0}-x^{*}\right)\right)\left(x_{0}-x^{*}\right) d \theta .
$$


Notice that

$$
\left\|x^{*}+\theta\left(x_{0}-x^{*}\right)-x^{*}\right\|=\theta\left\|x_{0}-x^{*}\right\|<R,
$$

which shows that $x^{*}+\theta\left(x_{0}-x^{*}\right) \in U\left(x^{*}, R\right)$. In view of (11) and (17), we get that

$$
\left\|F^{\prime}\left(x^{*}\right)^{-1} F\left(x_{0}\right)\right\| \leq M\left\|x_{0}-x^{*}\right\| .
$$

Then using the first substep of the method for $n=0,(7),(10),(17)$ and (19), we get from

$$
x_{0}^{(1)}-x^{*}=\left(x_{0}-x^{*}-F^{\prime}\left(x_{0}\right)^{-1} F\left(x_{0}\right)\right)+(1+\alpha) F^{\prime}\left(x_{0}\right)^{-1} F\left(x_{0}\right)
$$

that

$$
\begin{aligned}
\left\|x_{0}^{(1)}-x^{*}\right\| \leq & \left\|x_{0}-x^{*}-F^{\prime}\left(x_{0}\right)^{-1} F\left(x_{0}\right)\right\|+|1+\alpha|\left\|F^{\prime}\left(x_{0}\right)^{-1} F\left(x_{0}\right)\right\| \\
\leq & \left\|F^{\prime}\left(x_{0}\right)^{-1} F^{\prime}\left(x^{*}\right)\right\| \quad\left\|\int_{0}^{1} F^{\prime}\left(x^{*}\right)^{-1}\left(F^{\prime}\left(x^{*}+\theta\left(x_{0}-x^{*}\right)\right)-F^{\prime}\left(x_{0}\right)\right)\left(x_{0}-x^{*}\right) d \theta\right\| \\
& +|1+\alpha| \quad\left\|F^{\prime}\left(x_{0}\right)^{-1} F^{\prime}\left(x^{*}\right)\right\| \quad\left\|F^{\prime}\left(x^{*}\right)^{-1} F\left(x_{0}\right)\right\| \\
\leq & \frac{L\left\|x_{0}-x^{*}\right\|^{2}}{2\left(1-L_{0}|| x_{0}-x^{*} \|\right)}+|1+\alpha| \frac{M\left\|x_{0}-x^{*}\right\|}{1-L_{0}\left\|x_{0}-x^{*}\right\|} \\
= & g_{1}\left(\left\|x_{0}-x^{*}\right\|\right)\left\|x_{0}-x^{*}\right\| \leq g_{1}(r) r=\tilde{r},
\end{aligned}
$$

which shows (13) for $i=1$ and $x_{0}^{(1)} \in U\left(x^{*}, \tilde{r}\right)$.

Now, using the second substep of the method for $n=0,(7),(10),(17)$ and (19) for $x_{0}=x_{0}^{(1)}$, we obtain from

$$
x_{0}^{(2)}-x^{*}=\left(x_{0}^{(1)}-x^{*}-F^{\prime}\left(x_{0}^{(1)}\right)^{-1} F\left(x_{0}^{(1)}\right)\right)+\left(F^{\prime}\left(x_{0}^{(1)}\right)^{-1}-F^{\prime}\left(x_{0}\right)^{-1}\right) F\left(x_{0}^{(1)}\right)
$$


that

$$
\begin{aligned}
\left\|x_{0}^{(2)}-x^{*}\right\| \leq & \left\|\left(x_{0}^{(1)}-x^{*}-F^{\prime}\left(x_{0}^{(1)}\right)^{-1} F\left(x_{0}^{(1)}\right)\right)\right\|+\left\|\left(F^{\prime}\left(x_{0}^{(1)}\right)^{-1}-F^{\prime}\left(x_{0}\right)^{-1}\right) F\left(x_{0}^{(1)}\right)\right\| \\
\leq & \left\|F\left(x_{0}^{(1)}\right)^{-1} F^{\prime}\left(x^{*}\right)\right\| \| \int_{0}^{1} F^{\prime}\left(x^{*}\right)^{-1}\left[F^{\prime}\left(x^{*}+\theta\left(x_{0}^{(1)}-x^{*}\right)\right)-F^{\prime}\left(x_{0}^{(1)}\right)\right]\left(x_{0}^{(1)}-x^{*}\right) d \theta \\
& +\left\|F^{\prime}\left(x_{0}^{(1)}\right)^{-1} F^{\prime}\left(x^{*}\right)\right\|\left[\left\|F^{\prime}\left(x^{*}\right)^{-1}\left(F^{\prime}\left(x_{0}\right)-F^{\prime}\left(x^{*}\right)\right)\right\|+\left\|F^{\prime}\left(x^{*}\right)^{-1}\left(F^{\prime}\left(x^{*}\right)-F^{\prime}\left(x_{0}^{(1)}\right)\right)\right\|\right] \\
& \left\|F^{\prime}\left(x_{0}^{(1)}\right)^{-1} F^{\prime}\left(x^{*}\right)\right\|\left\|F^{\prime}\left(x^{*}\right)^{-1} F\left(x_{0}^{(1)}\right)\right\| \\
\leq & \frac{L\left\|x_{0}^{(1)}-x^{*}\right\|^{2}}{2\left(1-L_{0}\left\|x_{0}^{(1)}-x^{*}\right\|\right)}+\frac{L_{0}\left(\left\|x_{0}^{(1)}-x^{*}\right\|+\left\|x_{0}-x^{*}\right\|\right) M\left\|x_{0}^{(1)}-x^{*}\right\|}{\left(1-L_{0}\left\|x_{0}-x^{*}\right\|\right)\left(1-L_{0}\left\|x_{0}^{(1)}-x^{*}\right\|\right)} \\
\leq & \frac{L\left(g_{1}\left(\left\|x_{0}-x^{*}\right\|\right)\right)^{2}\left\|x_{0}-x^{*}\right\|^{2}}{2\left(1-L_{0} g_{1}\left(\left\|x_{0}-x^{*}\right\|\right)\left\|x_{0}-x^{*}\right\|\right)} \\
& +\frac{L_{0} M\left(1+g_{1}\left(\left\|x_{0}-x^{*}\right\|\right)\right) g_{1}\left(\left\|x_{0}-x^{*}\right\|\right)\left\|x_{0}-x^{*}\right\|^{2}}{\left(1-L_{0}\left\|x_{0}-x^{*}\right\|\right)\left(1-L_{0} g_{1}\left(\left\|x_{0}-x^{*}\right\|\right)\left\|x_{0}-x^{*}\right\|\right)} \\
\leq & {\left[\frac{L\left(g_{1}\left(\left\|x_{0}-x^{*}\right\|\right)\right)}{2\left(1-L_{0} g_{1}\left(\left\|x_{0}-x^{*}\right\|\right)\left\|x_{0}-x^{*}\right\|\right)}\right.} \\
& \left.+\frac{L_{0} M\left(1+g_{1}\left(\left\|x_{0}-x^{*}\right\|\right)\right)}{\left(1-L_{0}\left\|x_{0}-x^{*}\right\|\right)\left(1-L_{0} g_{1}\left(\left\|x_{0}-x^{*}\right\|\right)\left\|x_{0}-x^{*}\right\|\right)}\right] g_{1}\left(\left\|x_{0}-x^{*}\right\|\right)\left\|x_{0}-x^{*}\right\|^{2} \\
& =g_{2}\left(\left\|x_{0}-x^{*}\right\|\right)\left\|x_{0}-x^{*}\right\|<\left\|x_{0}-x^{*}\right\|<r
\end{aligned}
$$

which shows (14) for $n=2$ and $x_{0}^{(2)} \in U\left(x^{*}, r\right)$.

We obtain using induction

$$
\left\|x_{0}^{(i)}-x^{*}\right\| \leq g_{i}\left(\left\|x_{0}-x^{*}\right\|\right)\left\|x_{0}-x^{*}\right\|<\left\|x_{0}-x^{*}\right\|<r,
$$

which shows (14) for $i=2,3, \ldots, k-1$. and (15).

Similarly, from the identity

$$
\begin{aligned}
x_{1}^{(1)}-x^{*} & =x_{1}-x^{*}+\alpha F^{\prime}\left(x_{1}\right)^{-1} F\left(x_{1}\right) \\
& =\left(x_{1}-x^{*}-F^{\prime}\left(x_{1}\right)^{-1} F\left(x_{1}\right)\right)+(1+\alpha) F^{\prime}\left(x_{1}\right)^{-1} F\left(x_{1}\right)
\end{aligned}
$$

we get in turn

$$
\left\|x_{1}^{(1)}-x^{*}\right\| \leq g_{1}\left(\left\|x_{1}-x^{*}\right\|\right)\left\|x_{1}-x^{*}\right\|<\tilde{r},
$$

and

$$
\left\|x_{1}^{(2)}-x^{*}\right\| \leq g_{2}\left(\| x_{1}-x^{*}||\right)\left\|x_{1}-x^{*}\right\|<\left\|x_{1}-x^{*}\right\|<r .
$$

In this way, we arrive at

$$
\left\|x_{1}^{(i)}-x^{*}\right\| \leq g_{i}\left(\left\|x_{1}-x^{*}\right\|\right)|| x_{1}-x^{*}\|<\| x_{1}-x^{*} \|<r,
$$


$i=2,3, \ldots, k-1$ and

$$
\left\|x_{1}^{(k)}-x^{*}\right\| \leq g_{k}\left(\left\|x_{1}-x^{*}\right\|\right)\left\|x_{1}-x^{*}\right\|<\left\|x_{1}-x^{*}\right\|<r .
$$

By simply replacing $x_{0}, x_{0}^{(i)}, x_{1}$ by $x_{m}, x_{m}^{(i)}, x_{m+1}$, in the preceding estimates we complete the induction. Then, from the estimate

$$
\left\|x_{n+1}-x^{*}\right\|<\left\|x_{n}-x^{*}\right\|<r
$$

we deduce that

$$
\lim _{n \rightarrow \infty} x_{n}=x^{*}
$$

and $x_{n+1} \in U\left(x^{*}, R\right)$. The uniqueness part follows classical arguments [6].

As noted previously, method (3) has convergence order $k+1$, with $k \geq 2$. The convergence order was established in [2] using Taylor's expansions and hypotheses requiring the existence of at least the third Fréchet derivative. These hypotheses limit the applicability of method (3). As a motivational example for Theorem 1, let us define the function $F$ for $X=Y=\mathbb{R}, D=\left[-\frac{5}{2}, \frac{5}{2}\right]$ by

$$
F(x)= \begin{cases}x^{3} \ln x^{2}-x^{4}+x^{5} & x \neq 0 \\ 0 & x=0\end{cases}
$$

Then, we have that

$$
\begin{aligned}
F^{\prime}(x) & =3 x^{2} \ln x^{2}+5 x^{4}-4 x^{3}+2 x^{2} \\
F^{\prime \prime}(x) & =6 x \ln x^{2}+20 x^{3}-12 x^{2}+10 x, \\
F^{\prime \prime \prime}(x) & =6 \ln x^{2}+60 x^{2}-24 x+22 .
\end{aligned}
$$

The results in [2] cannot be applied, since $F^{\prime \prime \prime}(x)$ is not bounded on $D$. However, Theorem 1 can be applied with say $L_{0}=L=96.66$ and $M=2$.

Moreover, results using Taylor's expansions as in [2] suggest that the initial guess should be close to the solution $x^{*}$. But, how close initial guess should be for the convergence of method (3) to $x^{*}$. On the other hand, how do we compute the error bounds $\left\|x_{n}-x^{*}\right\|$ in this case. These concerns are addressed in Theorem 1, since computable estimates on the radius of convergence as well as the error bounds are provided.

\subsection{Analysis of the efficiency}

In this section, we use the efficiency index, $(E I)$, and the computational efficiency, $(C E)$, to compare different iterative methods. These are classical measurements of the cost of 


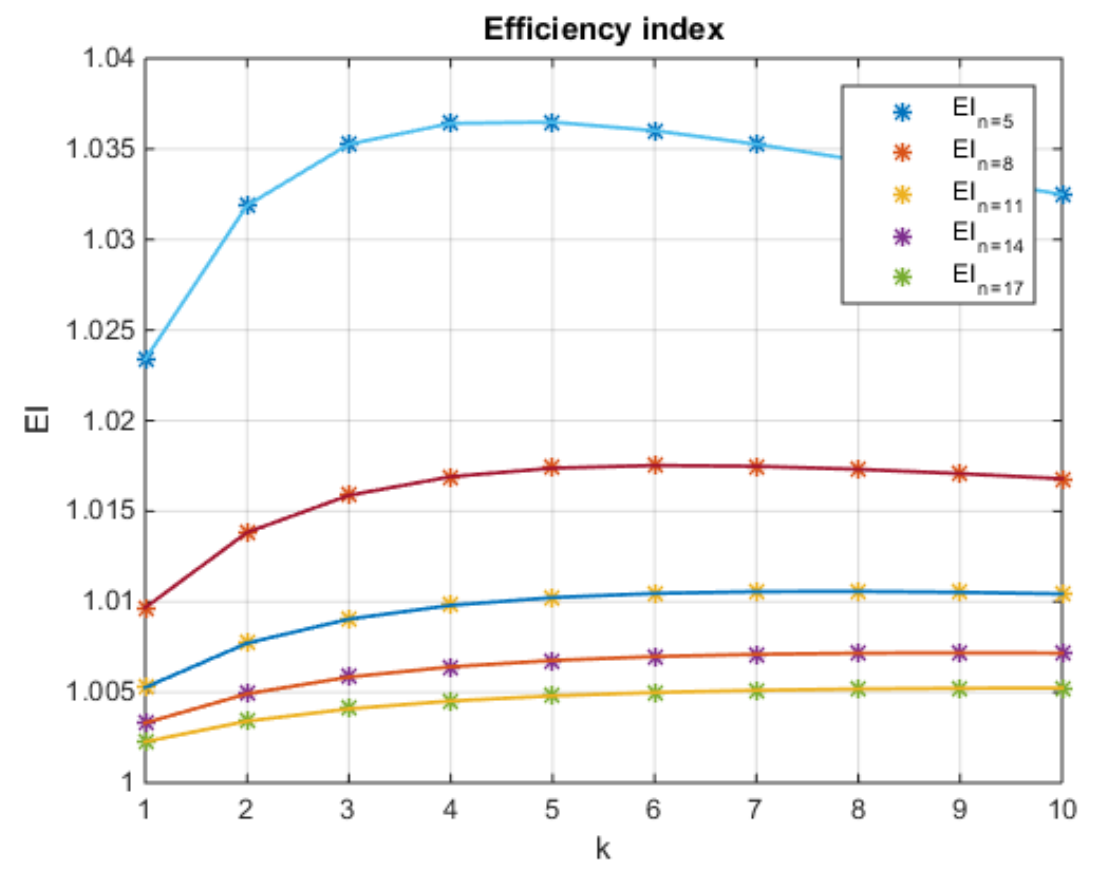

Figure 1: Efficiency index for $k=1: 10$ and $n=5: 3: 17$

applying a method, due respectively to Traub [24] and Ostrowski [23]. They are defined in the following way:

$$
E I=\rho^{1 / a} \quad \text { and } \quad C E=\rho^{1 / p},
$$

where $\rho$ is the order of convergence of the method, a represents the number of the evaluations of functions necessary to apply the method and $p$ is the number of operations (products and divisions) that are needed to compute each iteration of the method.

Then, in the presented multi-step method (3), we perform a new function evaluation in each step, so the value of $E I$ in function of $k$, the steps performed, and $n$, the size problem, is:

$$
E I(k, n)=(k+1) \frac{1}{n^{2}+k n}
$$

The $C E$ index is given by the number of products and quotients that we need for solving $k$ linear systems with the same matrix of coefficient, by using $L U$ factorization. We have:

$$
E C(k, n)=(k+1) \frac{3}{n^{3}+3 k n^{2}-n}
$$

In Figures 1 and 2 we can see these values for different methods by taking values for $k$ from 1 to 10 and considering problems of different sizes, $n=5,8,11,14,17$. We observe a general behavior in both indices, that is, when the size of the problem increases obviously 


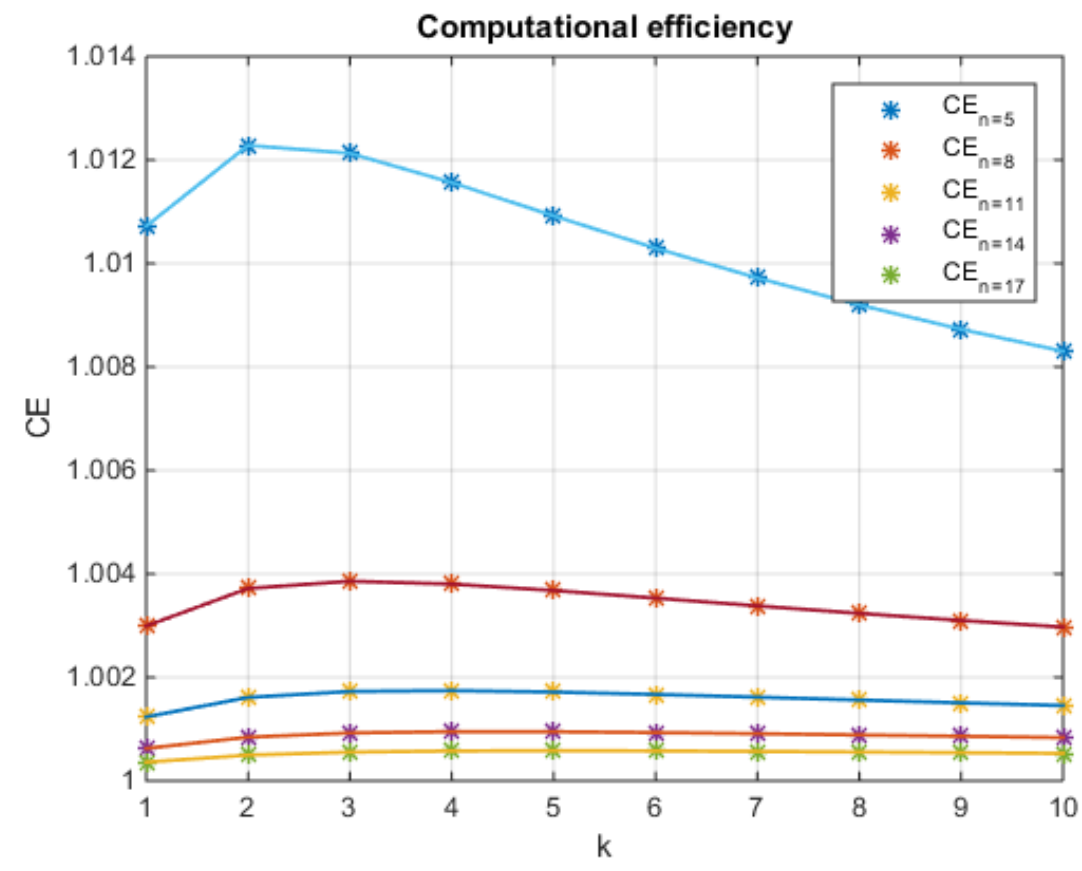

Figure 2: Computational efficiency for $k=1: 10$ and $n=5: 3: 17$

the efficiency decreases and tends to 1 . On the other hand, by considering increasing values of $k$ the efficiency indices increase until reaching a maximum from which the efficiency drops, this trend is more pronounced in the efficiency index than in the computational efficiency. In the efficiency index the average value for this maximum is about 5 but or 6 in the computational efficiency is about 3 or 4 . Notice that the method corresponding to $k=1$ is Newton's method, the $E I$ for other values of $k$ and $n$ is always better. Moreover, for $C E$, we can find worse behavior than Newton's method only for small size systems, in case $n=5$ and $k \geq 7$.

\section{$2.2 \quad$ A particular example}

In this subsection, a numerical example is worked out to demonstrate the application of our local convergence analysis for the k-steps iterative method described by (3).

We consider a nonlinear integral equation of Hammerstein type. These equations have many applications, such that, problems of electro-magnetic fluid dynamics, in the kinetic theory of gases, and in reformulation of boundary value problems, etc. These equations are of the form:

$$
x(s)=u(s)+\int_{l}^{m} K(s, t) H(x(t)) d t, \quad l \leq s \leq m,
$$

for $x(s), u(s) \in C[l, m]$ (the space of continuous functions in $[l, m]$ ) with $-\infty<l<m<$ 
$\infty, K$ is the kernel function and $H$, is a polynomial function. The usual technique to solve this kind of equations consists in expressing them as a nonlinear operator in a Banach space. That is

$$
\begin{gathered}
F(x)=0 \\
{[F(x)](s)=x(s)-u(s)-\int_{l}^{m} K(s, t) H(x(t)) d t}
\end{gathered}
$$

where $F: D \subseteq C[l, m] \rightarrow C[l, m]$ with $D$ a non-empty open convex subset.

We use the uniform norm $\|\nu\|=\max _{s \in[l, m]}|\nu(s)|$.

Next, we apply our theoretical study presented in Theorem 1 to a particular Hammerstein equation given by:

$$
F(x(s))=x(s)-\frac{21}{22} s-\frac{3 s}{11} \int_{0}^{1}(1+t)\left(1+x(t)^{2}\right) d t,
$$

with $x(s)$ in $\mathcal{C}[0,1]$.

The numerical scheme of this equation follows by applying a quadrature formula with $n$ nodes $t_{i}$ and their corresponding weights $a_{i}$ :

$$
\int_{0}^{1} \xi(t) d t \approx \sum_{i=1}^{n} a_{i} \xi\left(t_{i}\right)
$$

If we denote $x\left(t_{i}\right)=x_{i}, i=1,2, \ldots, n$, we transform the equation (21) into the following nonlinear system:

$$
x_{i}-\frac{21}{22} t_{i}-\frac{3 t_{i}}{11} \sum_{i=1}^{n} a_{i}\left(1+t_{i}\right)\left(1+x_{i}^{2}\right)=0,
$$

Obviously this system can be solved by applying the proposed iterative method given by (3). But for our local study we obtain the derivative of (21) that can be given by

$$
F^{\prime}(x(s))(v(s))=v(s)-\frac{3 s}{11} \int_{0}^{1}(1+t) 2 x(t) v(s) d t .
$$

So, for the solution of $x^{*}(s)=2 s$ we obtain $L=L_{0}=9$ and $M=2$. Now using the iterative method (3) by taking $k=4$, that correspond to an efficient method as we have deduced in section (2.1), and for $\alpha=-1$, we get:

$$
r_{\bar{p}_{1}}=0.081339>r_{\bar{p}_{2}}=0.055216>r_{\bar{p}_{3}}=0.044616>r_{\bar{p}_{4}}=0.039438 .
$$

The zeros for functions $g_{i}$ are:

$$
r_{\bar{g}_{2}}=0.048076<r_{\bar{g}_{3}}=0.040142<r_{\bar{g}_{4}}=0.036622
$$


so $r=0.036622$ and $\tilde{r}=0.009002395101316$. Each function of the ball $U(2 s, 0.036622)$ can be taken as a starting function in order to obtain the convergence for the nonlinear problem (21). The obtained solution is unique in $U(2 s, 0.222222) \bigcap D$.

By choosing $\alpha=1$, we obtain the following radii:

$$
r_{\bar{p}_{1}}=0.021795>r_{\bar{p}_{2}}=0.010536>r_{\bar{p}_{3}}=0.007895>r_{\bar{p}_{4}}=0.006985
$$

while the zeros for functions $g_{i}$ are:

$$
r_{\bar{g}_{2}}=0.002031<r_{\bar{g}_{3}}=0.004422<r_{\bar{g}_{4}}=0.005463
$$

and then $r=0.002031$ and $\tilde{r}=0.008293$. So, in this case the ball $U(2 s, 0.002031)$ provides starting functions for the problem (21). In Figures 3 and 4 can be seen the graphics of functions $\bar{p}$ and $\bar{g}$ that provide the local convergence radius.

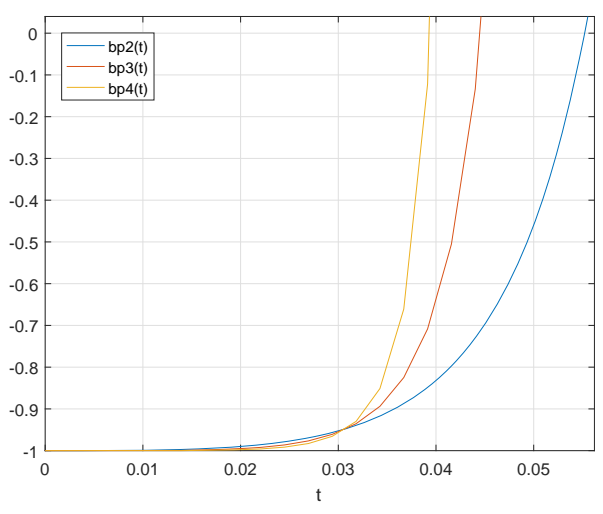

Figure 3: Functions $\bar{p}_{i}(t)$, for $\alpha=-1$.

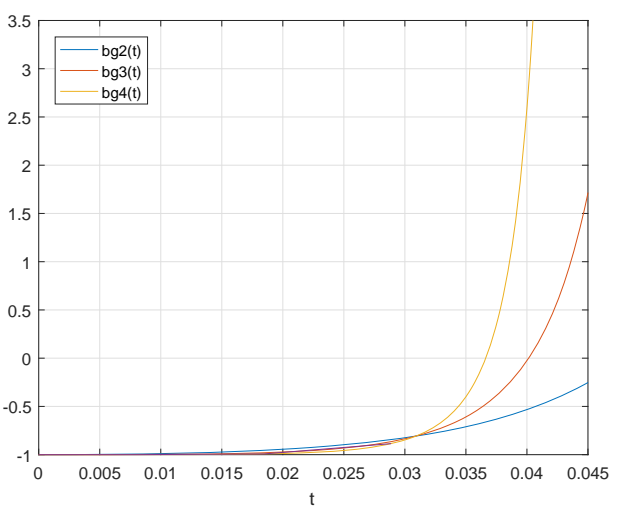

Figure 4: Functions $\bar{g}_{i}(t)$ for $\alpha=-1$.

The comparison of radii of convergence balls of our iterative method (3) for different values of parameters $\alpha$ and $k=4$ is displayed in Table 1 .

Table 1: Radii of convergence balls for different values of $\alpha$ with four steps

\begin{tabular}{|c|c|c|c|c|}
\hline$\alpha>0$ & $\alpha=0.85$ & $\alpha=0.95$ & $\alpha=1.15$ & $\alpha=1.25$ \\
\hline $\mathbf{r}$ & 0.002313 & 0.002119 & 0.001796 & 0.001662 \\
\hline$r_{1}$ & 0.0087649 & 0.0084447 & 0.007866 & 0.0076056 \\
\hline$\alpha<0$ & $\alpha=-0.85$ & $\alpha=-0.95$ & $\alpha=-1.15$ & $\alpha=-1.25$ \\
\hline $\mathbf{r}$ & 0.027189 & 0.031981 & 0.027189 & 0.021489 \\
\hline$r_{1}$ & 0.0152038 & 0.0109536 & 0.0152037 & 0.0158971 \\
\hline
\end{tabular}

The best radius is obtained for $\alpha=-1$, that correspond with taking Newton's method in first step. On the other hand, from Table 1, we observe that the radius is always better for negative values near to alpha $=-1$. Finally, we notice that, for all tested cases, $r_{1}>r$ for $\alpha>0$ and $r_{1}<r$ for $\alpha<0$. Obviously if we perform less steps in our method the radii of the local convergence balls will increase. 


\section{References}

[1] Ahmad, F., Tohidi, E., Carrasco, J. A., A parameterized multi-step Newton method for solving systems of nonlinear equations. Numer. Algorithms 71 (3) (2016) 631-653.

[2] Amat S., Bermúdez C., Busquier S., Plaza S., On two families of high order Newton type methods, Appl. Math. Let. 25 (2012) 2209-2217.

[3] Amat S., Bermúdez C., Hernández-Verón M., Martínez E., On an efficient k-step iterative method for nonlinear equations, J. Comp.Appl. Math. 302 (15) (2016) 258271.

[4] Amat, S., Busquier, S., Bermúdez, C., Magreñán, Á.A., On the election of the damped parameter of a two-step relaxed Newton-type method. Nonlinear Dynam. 84 (1) (2016) 9-18.

[5] Amat S., Busquier S., Grau A., Grau-Sánchez M., Maximum efficiency for a family of Newton-like methods with frozen derivatives and some applications, Appl. Math. Comp. 219 (2013) 7954-7963.

[6] Argyros I.K., George S., Local convergence for some high convergence order Newtonlike methods with frozen derivatives SeMA J. 70 (1) (2015) 47-59.

[7] Argyros, I.K., George, S., On the semilocal convergence of a two step Newton method under the $\gamma$-condition. J. Nonlinear Anal. Optim. 6 (2) (2015) 73-84.

[8] Argyros, Ioannis K.; George, Santhosh Convergence analysis of a three step Newtonlike method for nonlinear equations in Banach space under weak conditions. An. Univ. Vest Timiş. Ser. Mat.-Inform. 54 (2) (2016) 37-46.

[9] Argyros, I.K., George, S., Local convergence of a two-step Newton-secant method for equations with solutions of multiplicity greater than one. Pan Amer. Math. J. 27 (1) (2017) 15-28.

[10] Argyros, I.K., Magreñán, Á.A. Local convergence and the dynamics of a two-step Newton-like method. Internat. J. Bifur. Chaos Appl. Sci. Engrg. 26 (5) (2016) 18 pp.

[11] Argyros, I.K., Magreñán, Á.A., Orcos, L., Sicilia, J.A., Local convergence of a relaxed two-step Newton like method with applications. J. Math. Chem. 55(7) (2017) 14271442.

[12] Ha-Duong T., Jaoua M., Menif F., A modified frozen Newton method to identify a cavity by means of boundary measurements, Math. Comput. Simulat. 66 (4-5) (2004) 355-366. 
[13] Hernández-Verón, M. A., Martínez, E., On the semilocal convergence of a three steps Newton-type iterative process under mild convergence conditions. Numer. Algorithms 70 (2) (2015) 377-392.

[14] Hinze M., Kunisch K., Second order methods for optimal control of time-dependent fluid flow, SIAM J. Contr. Optim. 40-3 (2001) 925-946.

[15] Jin Q., On a class of frozen regularized Gauss-Newton methods for nonlinear inverse problems, Math. Comput. 79-272 (2010) 2191-2211.

[16] Kantorovich L.V., Akilov G.P., Functional analysis Pergamon Press, Oxford, 1982.

[17] Laumen M., Newton's method for a class of optimal shape design problems, SIAM J. Optim. 10 (2000) 503-533.

[18] Li, Y., Guo, X-P., Multi-step modified Newton-HSS methods for systems of nonlinear equations with positive definite Jacobian matrices. Numer. Algorithms 75 (1) (2017) $55-80$.

[19] Ma C., Chen X., The convergence of a one-step smoothing Newton method for P0NCP based on a new smoothing NCP-function, J. Comp. Appl. Math. 216 (2008) $1-13$.

[20] Mahale P., Nair M.T., A simplified generalized Gauss-Newton method for nonlinear ill-posed problems, Math. Comput. 78-265 (2009) 171-184.

[21] Moghrabi, I. A. R., Implicit extra-update multi-step quasi-Newton methods. Int. J. Oper. Res. 28 (2) (2017) 216-228.

[22] Petkovic M.S., Neta B., Petkovic L., Džunič J., Multipoint methods for solving nonlinear equations, Elsevier, 2013.

[23] Ostrowski, A.M., Solutions of Equations in Euclidean and Banach Spaces. Academic Press, New York (1973).

[24] Traub, J.F., Iterative Methods for the Solution of Equations. Prentice-Hall, Englewood Cliffs, New Jersey (1964).

[25] I.K. Argyros, S. George, Local convergence of modified Halley-like methods with less computation of inversion, Novi Sad J. Math, Draft version, (2015). 